\title{
SYNTHESIS, ANTIBACTERIAL AND ANTIFUNGAL ACTIVITY OF SOME NEW 1,3,4-OXADIAZOLES AND 2-SUBSTITUTED AMINO-1,3,4-OXADIAZOLE DERIVATIVES CONTAINING BENZIMIDAZOLE MOIETY
}

\author{
F.A. Ashour ${ }^{1}$, S.A.M. El-Hawash, M.A. Mahran, A.H. Yousry ${ }^{*}$ and A.F. Hamouda* \\ Pharmaceutical Chemistry Department, Faculty of Pharmacy, Alexandria University, Egypt \\ * Department of Microbiology, High Institute of Public Health, University of Alexandria, Egypt

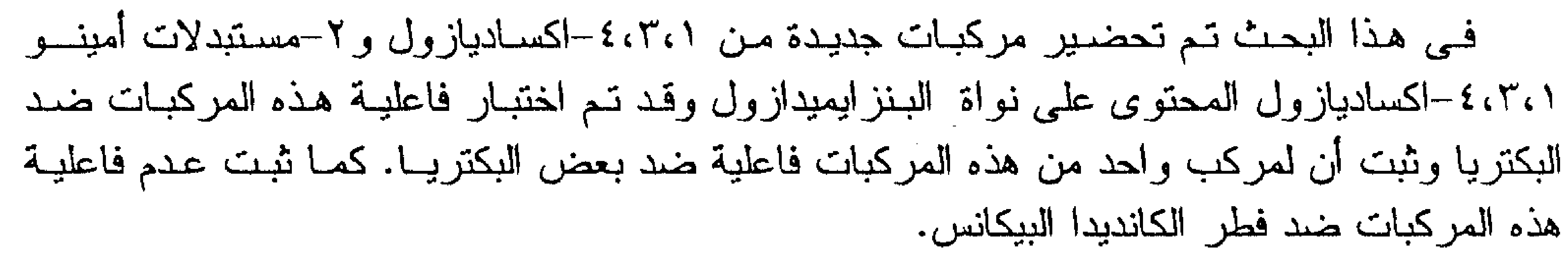

New 2-[4-(3-acetyl-2-substituted-2,3-dihydro-1,3,4-oxadiazol-5-yl)phenoxymethyl]-1Hbezimidazoles (V) and 2-[4-(3-acetyl-2-substituted-2,3-diydro-1,3,4-oxadiazol5yl)phenoxymetyl]-1-methyl-1H-benzimidazoles (VI) were synthesized by cyclization of 2-[4alkylidene or arylidenehydrazinocarbonyl)phenoxymethyl]-1H-benzimidazoles (III) or 2-[4(alkylidene or arylidenehydrazinocarbonyl)phenoxymethyl]-1-methyl-1H-benzimidazoles (IV) with acetic anhydride.

New series of 2-[4-substitutedamino-1,3,4-oxadiazol-5-yl)phenoxymethyl]-1Hbenzimidazoles (IX) and 2-(4-substitutedamino-1,3,4-oxadiazol-5-yl)phenoxymethyl]-1-methyl1 1H-benzimidazoles $(X)$ were obtained by cyclodesulfurization of 2-[4-(substitutedthiocarbamoylhydrazinocarbonyl)phenoxymethyl]-1H-benzimidazoles (VII) or 2-[4(substitutedthiocarbamoylhydrazinocarbonyl)phenoxymethyl-1-methyl-1H-benzimidazoles (VIII). The structure of the newly synthesized compounds were elucidated by elemental analysis, $I R$ and ${ }^{\prime} H-N M R$ spectra. Their antimicrobial activity was studied.

\section{INTRODUCTION}

Various compounds containing 1,3,4oxadiazole moiety have been reported to exhibit antibacterial $^{1-3}$ and antifungal ${ }^{3-6}$ activity. In addition, antibacterial ${ }^{7,8}$ and antifungal ${ }^{8-10}$ activities have been ascribed to several derivatives of benzimidazoles.

As a continuation of our research on the synthesis of oxadiazoles as antimicrobial agents $^{11}$, it was designed in the present investigation to synthesize new series of compounds containing an oxadiazole moiety attached to benzimidazole and evaluate their antibacterial and antifungal activities.

\section{EXPERIMENTAL}

Melting points were determined in open capillaries and are uncorrected. IR spectra were recorded as nujol mulls on a Shimadzu IR 408 spectrometer. ${ }^{1} \mathrm{H}-\mathrm{NMR}$ spectra were recorded on JEOL FX90Q $90 \mathrm{MHz}$ spectrometer. Chemical shifts are reported in ppm. downfield from tetramethylsilane used as internal standard.Elemental analyses were carried out at the Microanalytical unit, University of Cairo, Egypt.

\footnotetext{
1 To whom correspondence should be addressed Received in 9/9/1993 \& Accepted in 28/11/1993
} 
4-(1-Methyl-1H-2-benzimidazolyl methyloxy) benzoic acid hydrazide (II):

A mixture of 2-[4-(ethoxycarbonyl) phenoxymethyl]-1-methyl-1H-benzimidazole ${ }^{12}$ $(0.3 \mathrm{~g}, 0.001 \mathrm{~mole})$ and hydrazine hydrate $(98 \%)(5 \mathrm{ml})$ was heated on a water bath for 3 hr. Water was then added and the precipitated solid was filtered, washed with water and crystallized from ethanol m.p. $217-18^{\circ} \mathrm{C}$; yield $(0.25 \mathrm{~g}, 80 \%)$. IR $\left(\mathrm{cm}^{-1}\right) 3400,3300,3205$ $\left(\mathrm{NH}_{2}\right.$ and $\left.\mathrm{NH}\right) ; 1675$ (amide I band); 1520 (amide II band); Anal. for $\mathrm{C}_{16} \mathrm{H}_{16} \mathrm{~N}_{4} \mathrm{O}_{2}$ Calcd. C:64.85, H: 5.44, N: 18.90, Found; C: 64.6, $\mathrm{H}: 5.4, \mathrm{~N}: 18.6$.

2-[4-(Alkylidene or arylidenehydrazinocarbonyl)phenoxymethyl]-1H-benzimidazoles (III a-f) and 2-[4-(alkylidene or arylidenehydrazinocarbonyl)phenoxymethyl]-1-methyl1 $H$-benzimidazoles (IV a-e):

To a solution of 0.01 mole of the acid hydrazide (I $)^{13}$ or (II) in ethanol $(50 \mathrm{ml})$ was added 0.01 mole of the appropriate aldehyde or ketone and 2 drops of glacial acetic acid. The reaction mixture was heated under reflux for 3-4 $\mathrm{hr}$ (compound III c,d and IV c needed $10 \mathrm{hr}$.), concentrated and cooled to room temperature. The resulting product was filtered (in case of III $c, d$ and IV $c$ the products deposited after addition of drops of water), dried and crystallized from ethanol or aqueous ethanol (Table 1). The IR $\left(\mathrm{cm}^{-1}\right)$ of compounds (III a-f) showed 3300-3200 (br. NH), 1660-1640 (C=O), compounds (IV a-e) showed 3250-3150 (NH); 1650-1645 $(\mathrm{C}=\mathrm{O}) .{ }^{1} \mathrm{H}-\mathrm{NMR}$ of compound (III b) $\left(\mathrm{DMSO}-\mathrm{d}_{6}\right) \delta \mathrm{ppm} ; 3.7\left(\mathrm{~s}, 3 \mathrm{H}, \mathrm{OCH}_{3}\right), 3.9$ $\left(\mathrm{s}, 3 \mathrm{H}, \mathrm{OCH}_{3}\right), 5.45\left(\mathrm{~s}, 2 \mathrm{H}, \underline{\mathrm{CH}}_{2}\right), 6.9-7.95(\mathrm{~m}$, $11 \mathrm{H}, \mathrm{Ar}-\underline{\mathrm{H}}), 8.4$ (br.s, 1H, NH) $11.8(\mathrm{~s}, 1 \mathrm{H}$, $\mathrm{NH}$ ). Compound (III f) ' $\mathrm{H}-\mathrm{NMR}\left(\mathrm{DMSO}-\mathrm{d}_{6}\right) \delta$ ppm; $2.3\left(\mathrm{~s}, 3 \mathrm{H}, \mathrm{CH}_{3}\right), 5.35\left(\mathrm{~s}, 2 \mathrm{H}, \mathrm{CH}_{2}\right)$, 7.05-7.85 (m, 12H, Ar- $\underline{\mathrm{H}}), 10.55(\mathrm{~s}, 1 \mathrm{H}, \mathrm{N} \underline{\mathrm{H}})$, $12.6\left(\mathrm{~s}, 1 \mathrm{H}, \mathrm{N} \underline{\mathrm{H}}\right.$ ). Compound (IV d) ${ }^{1} \mathrm{H}-\mathrm{NMR}$ $\left(\right.$ DMSO-d $\left._{6}\right) \delta$ ppm; $2.35\left(\mathrm{~s}, 6 \mathrm{H}, 2 \mathrm{CH}_{3}\right), 3.9$ (s, $\left.3 \mathrm{H}, \mathrm{N}-\mathrm{CH}_{3}\right), 5.5\left(\mathrm{~s}, 2 \mathrm{H}, \mathrm{CH}_{2}\right), 7.17-7.95(\mathrm{~m}$, $12 \mathrm{H}, \operatorname{Ar}-\underline{\mathrm{H}}), 10.43(\mathrm{~s}, 1 \mathrm{H}, \mathrm{O}=\mathrm{C}-\mathrm{N} \underline{\mathrm{H}})$.
2-[4-(3-Acetyl-2-substituted-2,3-dihydro-1,3,4oxadiazol-5-yl)phenoxymethyl]-1 $H$-benzimidazoles (V a-f) and 2-[4-(3-acetyl-2substituted-2,3-dihydro-1,3,4-oxadiazol-5yl)phenoxymethyl]-1-methyl-1 $H$-benzimidazoles (VI a-e).

A mixture of the appropriate III a-f, or IV a-e $(0.001$ mole) and acetic anhydride $(5 \mathrm{ml})$ was heated under reflux for $2 \mathrm{hr}$. The reaction mixture was concentrated, cooled, and the solid separated after addition of cold water was filtered, washed with water, dried and crystallized from the proper solvent (Table 2). The IR $\left(\mathrm{cm}^{-1}\right)$ of compounds ( $\mathrm{V}$ a-f) showed 3200-3150 (NH), 1700-1680 (C=O); Compounds (VI a-e) showed 1670-1660 $(\mathrm{C}=\mathrm{O})$, ${ }^{1} \mathrm{H}-\mathrm{NMR}$ of compound $\mathrm{V} \mathrm{f}\left(\mathrm{DMSO}-\mathrm{d}_{6}\right) \delta \mathrm{ppm}$, $1.92\left(\mathrm{~s}, 3 \mathrm{H}, \mathrm{CH}_{3}\right), 2.56\left(\mathrm{~s}, 3 \mathrm{H}, \mathrm{COCH}_{3}\right), 5.52$ (s, $\left.2 \mathrm{H}, \mathrm{CH}_{2}\right), 7.36-8.24(\mathrm{~m}, 12 \mathrm{H}, \mathrm{Ar}-\underline{\mathrm{H}}) ; 10.24$ $(\mathrm{s}, 1 \mathrm{H}, \mathrm{N} \underline{\mathrm{H}})$. Compound VI d $\left(\mathrm{CDCl}_{3}\right) \delta \mathrm{ppm}$ : $2.4\left(\mathrm{~s}, 3 \mathrm{H}, \mathrm{CH}_{3}\right), 2.45\left(\mathrm{~s}, 3 \mathrm{H}, \mathrm{C}_{6} \mathrm{H}_{4}-\mathrm{CH}_{3}\right), 2.5$ $\left(\mathrm{s}, 3 \mathrm{H}, \mathrm{COCH}{ }_{3}\right), 3.8\left(\mathrm{~s}, 3 \mathrm{H}, \mathrm{N}-\mathrm{CH}_{3}\right), 5.4(\mathrm{~s}, 2 \mathrm{H}$. $\left.\underline{\mathrm{CH}}_{2}\right), 7.0-7.9(\mathrm{~m}, 12 \mathrm{H}, \mathrm{Ar}-\underline{\mathrm{H}})$.

2-[4-(Substitutedthiocarbamoylhydrazinocarbonyl)phenoxymethyl]-1-methyl $1 \mathrm{H}$ benzimidazoles (VIII a-e).

Equimolar quantities of 4-(1-methyl-1Hbenzimidazole-2-yl) methoxybenzoic acid hydrazide $(0.01$ mole $)$ and the appropriate isothiocyanate $(0.01 \mathrm{~mole})$ were heated under reflux in ethanol $(100 \mathrm{ml})$ for $1-8$ hours. The excess ethanol was distilled and the crystalline solid, separated on cooling was collected and crystallized from the proper solvent (Table 3). The IR $\left(\mathrm{cm}^{-1}\right)$ of compounds (VIII a-e) showed 3200-3100 (NH); 1675-1670 (C=O); 1545$1540,1335-1330,1145-1140,940-930(\mathrm{~N}-\mathrm{C}=\mathrm{S}$ amide I, II, III and IV bands). ${ }^{1} \mathrm{H}-\mathrm{NMR}$ of compound VIII e (DMSO- $\left.\mathrm{d}_{6}\right) \delta \mathrm{ppm}$ showed $\left.2.329 \mathrm{~s}, 3 \mathrm{H}, \mathrm{C}_{6} \mathrm{H}_{4}-\mathrm{CH}_{3}\right), 3.92\left(\mathrm{~s}, 3 \mathrm{H}, \mathrm{N}-\mathrm{CH}_{3}\right)$, $5.68\left(\mathrm{~s}, 2 \mathrm{H}, \mathrm{CH}_{2}\right), 7.28-8.28(\mathrm{~m}, 12 \mathrm{H}, \mathrm{Ar}-\mathrm{H})$, $9.92, \quad 10.08, \quad 10.72 \quad(3 \mathrm{~s}, \quad 3 \mathrm{H}, \quad \mathrm{NH}$ of thiosemicarbazide moiety, deuterium exchangeable). 
Table 1: $\quad$ 2-[4-(Alkylidene or arylidenehydrazinocarbonyl)phenoxymethyl]-1H-benzimidazoles (III a-f) and 2-[4-(alkyidene or arylidenehydrazinocarbonyl)phenoxymthyl]-1-methyl-1 $H$ benzimidazoles (IV a-e).

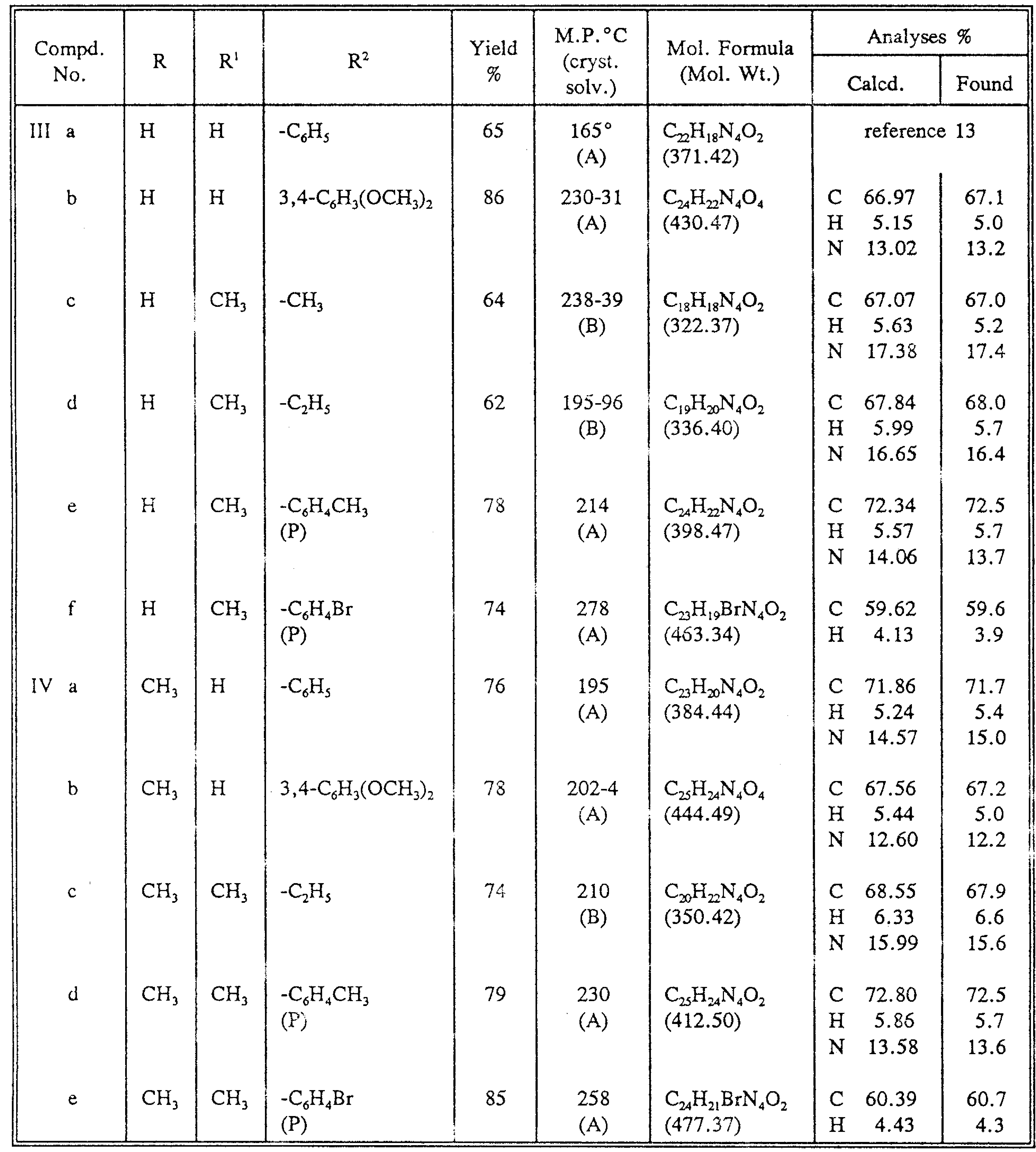

$A=$ Ethanol,

$\mathrm{B}=$ Ethanol $/ \mathrm{H}_{2} \mathrm{O}$. 
Table 2: 2-[4-(3-Acetyl-2-substituted-2,3-dihydro-1,3,4-oxadiazol-5-yl)phenoxymethyl]-1Hbenzimidazoles (V a-f) and 2-[4-(3-acetyl-2-substituted-2,3-dihydro-1,3,4-oxadiazol-5yl)phenoxymethyl]-1-methyl-1H-benzimidaziles (VI a-e).

\begin{tabular}{|c|c|c|c|c|c|c|c|c|}
\hline \multirow{2}{*}{$\begin{array}{c}\text { Compd. } \\
\text { No. }\end{array}$} & \multirow{2}{*}{$\mathrm{R}$} & \multirow{2}{*}{$\mathrm{R}^{\prime}$} & \multirow{2}{*}{$\mathrm{R}^{2}$} & \multirow{2}{*}{$\begin{array}{c}\text { Yield } \\
\%\end{array}$} & \multirow{2}{*}{$\begin{array}{l}\text { M.P. }{ }^{\circ} \mathrm{C} \\
\text { (cryst. } \\
\text { solv.) }\end{array}$} & \multirow{2}{*}{$\begin{array}{l}\text { Mol. Formula } \\
\text { (Mol. Wt.) }\end{array}$} & \multicolumn{2}{|c|}{ Analyses \% } \\
\hline & & & & & & & Calcd. & Found \\
\hline V a & $\mathrm{H}$ & $\mathrm{H}$ & $-\mathrm{C}_{6} \mathrm{H}_{5}$ & 72 & $\begin{array}{c}152-53 \\
(\mathrm{~A})\end{array}$ & $\begin{array}{l}\mathrm{C}_{24} \mathrm{H}_{20} \mathrm{~N}_{4} \mathrm{O}_{3} \\
(412.45)\end{array}$ & $\begin{array}{cc}\mathrm{C} & 69.89 \\
\mathrm{H} & 4.89 \\
\mathrm{~N} & 13.58\end{array}$ & $\begin{array}{r}69.5 \\
4.9 \\
13.3\end{array}$ \\
\hline b & $\mathrm{H}$ & $\mathrm{H}$ & $3,4-\mathrm{C}_{6} \mathrm{H}_{3}\left(\mathrm{OCH}_{3}\right)_{2}$ & 76 & $\begin{array}{c}164-5 \\
(\mathrm{~A})\end{array}$ & $\begin{array}{l}\mathrm{C}_{26} \mathrm{H}_{24} \mathrm{~N}_{4} \mathrm{O}_{5} \\
(472.51)\end{array}$ & $\begin{array}{cc}\mathrm{C} & 66.09 \\
\mathrm{H} & 5.12 \\
\mathrm{~N} & 11.86\end{array}$ & $\begin{array}{r}55.9 \\
5.1 \\
11.5\end{array}$ \\
\hline c & $\mathrm{H}$ & $\mathrm{CH}_{3}$ & $-\mathrm{CH}_{3}$ & 68 & $\begin{array}{l}166-7 \\
\text { (B) }\end{array}$ & $\begin{array}{l}\mathrm{C}_{20} \mathrm{H}_{20} \mathrm{~N}_{4} \mathrm{O}_{3} \\
(364.41)\end{array}$ & $\begin{array}{cc}\mathrm{C} & 65.92 \\
\mathrm{H} & 5.53 \\
\mathrm{~N} & 15.37\end{array}$ & $\begin{array}{r}65.8 \\
5.3 \\
15.1\end{array}$ \\
\hline d & $\mathrm{H}$ & $\mathrm{CH}_{3}$ & $-\mathrm{C}_{2} \mathrm{H}_{5}$ & 65 & $\begin{array}{l}200 \\
\text { (B) }\end{array}$ & $\begin{array}{l}\mathrm{C}_{21} \mathrm{H}_{22} \mathrm{~N}_{4} \mathrm{O}_{3} \\
(378.43)\end{array}$ & $\begin{array}{cr}\mathrm{C} & 66.65 \\
\mathrm{H} & 5.86 \\
\mathrm{~N} & 14.80\end{array}$ & $\begin{array}{r}66.5 \\
5.9 \\
14.4\end{array}$ \\
\hline $\mathrm{e}$ & $\mathrm{H}$ & $\mathrm{CH}_{3}$ & $\begin{array}{l}-\mathrm{C}_{6} \mathrm{H}_{4} \mathrm{CH}_{3} \\
\text { (P) }\end{array}$ & 82 & $\begin{array}{l}190 \\
(\mathrm{~A})\end{array}$ & $\begin{array}{l}\mathrm{C}_{26} \mathrm{H}_{24} \mathrm{~N}_{4} \mathrm{O}_{3} \\
(440.51)\end{array}$ & $\begin{array}{cc}\mathrm{C} & 70.89 \\
\mathrm{H} & 5.49 \\
\mathrm{~N} & 12.72\end{array}$ & $\begin{array}{r}71.1 \\
5.3 \\
12.6\end{array}$ \\
\hline f & $\mathrm{H}$ & $\mathrm{CH}_{3}$ & $\begin{array}{l}-\mathrm{C}_{6} \mathrm{H}_{4} \mathrm{Br} \\
\text { (P) }\end{array}$ & 85 & $\begin{array}{l}250 \\
(\mathrm{~A})\end{array}$ & $\begin{array}{l}\mathrm{C}_{25} \mathrm{H}_{21} \mathrm{BrN}_{4} \mathrm{O}_{3} \\
(505.38)\end{array}$ & $\begin{array}{cc}\mathrm{C} & 59.42 \\
\mathrm{H} & 4.19 \\
\mathrm{~N} & 11.09\end{array}$ & $\begin{array}{r}59.3 \\
4.3 \\
10.6\end{array}$ \\
\hline IV a & $\mathrm{CH}_{3}$ & $\mathrm{H}$ & $-\mathrm{C}_{6} \mathrm{H}_{5}$ & 78 & $\begin{array}{l}157 \\
\text { (A) }\end{array}$ & $\begin{array}{l}\mathrm{C}_{25} \mathrm{H}_{22} \mathrm{~N}_{4} \mathrm{O}_{3} \\
(426.48)\end{array}$ & $\begin{array}{cc}\mathrm{C} & 70.41 \\
\mathrm{H} & 5.20 \\
\mathrm{~N} & 13.14\end{array}$ & $\begin{array}{r}70.7 \\
5.3 \\
13.2\end{array}$ \\
\hline b & $\mathrm{CH}_{3}$ & $\mathrm{H}$ & $3,4-\mathrm{C}_{6} \mathrm{H}_{3}\left(\mathrm{OCH}_{3}\right)_{2}$ & 75 & $\begin{array}{l}183 \\
(\mathrm{~A})\end{array}$ & $\begin{array}{l}\mathrm{C}_{27} \mathrm{H}_{26} \mathrm{~N}_{4} \mathrm{O}_{5} \\
(486.53)\end{array}$ & $\begin{array}{cr}\mathrm{C} & 66.66 \\
\mathrm{H} & 5.39 \\
\mathrm{~N} & 11.52\end{array}$ & $\begin{array}{r}66.5 \\
5.4 \\
11.1\end{array}$ \\
\hline c & $\mathrm{CH}_{3}$ & $\mathrm{CH}_{3}$ & $-\mathrm{C}_{2} \mathrm{H}_{5}$ & 70 & $\begin{array}{l}185 \\
\text { (B) }\end{array}$ & $\begin{array}{l}\mathrm{C}_{22} \mathrm{H}_{24} \mathrm{~N}_{4} \mathrm{O}_{3} \\
(392.46)\end{array}$ & $\begin{array}{lr}\mathrm{C} & 67.33 \\
\mathrm{H} & 6.16\end{array}$ & $\begin{array}{c}67.2 \\
6.1\end{array}$ \\
\hline d & $\mathrm{CH}_{3}$ & $\mathrm{CH}_{3}$ & $\begin{array}{l}-\mathrm{C}_{6} \mathrm{H}_{4} \mathrm{CH}_{3} \\
\text { (P) }\end{array}$ & 85 & $\begin{array}{l}165 \\
\text { (A) }\end{array}$ & $\begin{array}{l}\mathrm{C}_{27} \mathrm{H}_{26} \mathrm{~N}_{4} \mathrm{O}_{3} \\
(454.53)\end{array}$ & $\begin{array}{cr}\mathrm{C} & 71.35 \\
\mathrm{H} & 5.77 \\
\mathrm{~N} & 12.33\end{array}$ & $\begin{array}{r}71.7 \\
5.4 \\
12.6\end{array}$ \\
\hline $\mathrm{e}$ & $\mathrm{CH}_{3}$ & $\mathrm{CH}_{3}$ & $\begin{array}{l}-\mathrm{C}_{6} \mathrm{H}_{4} \mathrm{Br} \\
(\mathrm{P})\end{array}$ & 88 & $\begin{array}{l}95 \\
(\mathrm{~A})\end{array}$ & $\begin{array}{l}\mathrm{C}_{26} \mathrm{H}_{23} \mathrm{BrN}_{4} \mathrm{O}_{3} \\
(519.40)\end{array}$ & $\begin{array}{ll}\mathrm{N} & 10.79 \\
\mathrm{Br} & 15.38\end{array}$ & $\begin{array}{l}10.5 \\
15.4\end{array}$ \\
\hline
\end{tabular}

$\mathrm{A}=$ Ethanol $/ \mathrm{H}_{2} \mathrm{O}, \quad \mathrm{B}=\mathrm{CHCl}_{3} /$ Pet. ehter $40-60^{\circ} \mathrm{C}$. 
Table 3: $\quad$ 2-[4-(Substitutedthiocarbomoylhydrazinocarbonyl)phenoxymethyl]-1-methyl-1Hbenzimidazoles (VIII a-e).

\begin{tabular}{|c|c|c|c|c|c|c|}
\hline \multirow{2}{*}{$\begin{array}{c}\text { Compd. } \\
\text { No. }\end{array}$} & \multirow{2}{*}{$\mathrm{R}^{3}$} & \multirow{2}{*}{$\begin{array}{c}\text { Yield } \\
\%\end{array}$} & \multirow{2}{*}{$\begin{array}{l}\text { M.P. }{ }^{\circ} \mathrm{C} \\
\text { (cryst. } \\
\text { solv.) } \\
\end{array}$} & \multirow{2}{*}{$\begin{array}{c}\text { Mol. } \\
\text { Formula } \\
\text { (Mol. Wt.) }\end{array}$} & \multicolumn{2}{|c|}{ Analyses \% } \\
\hline & & & & & Calcd. & Found \\
\hline VIII $a$ & $\mathrm{n}-\mathrm{C}_{4} \mathrm{H}_{9}$ & 86 & $\begin{array}{c}222-24 \\
(\mathrm{~A})\end{array}$ & $\begin{array}{l}\mathrm{C}_{21} \mathrm{H}_{25} \mathrm{~N}_{2} \mathrm{O}_{2} \mathrm{~S} \\
(411.53)\end{array}$ & $\begin{array}{lc}\mathrm{C} & 61.29 \\
\mathrm{H} & 6.12 \\
\mathrm{~N} & 17.02 \\
\mathrm{~S} & 7.79\end{array}$ & $\begin{array}{r}61.40 \\
6.20 \\
17.20 \\
7.70\end{array}$ \\
\hline b & $\begin{array}{l}-\mathrm{C}_{6} \mathrm{H}_{11} \\
\text { (cyclo) }\end{array}$ & 95 & $\begin{array}{c}226-27 \\
(\mathrm{~A})\end{array}$ & $\begin{array}{l}\mathrm{C}_{23} \mathrm{H}_{27} \mathrm{~N}_{5} \mathrm{O}_{2} \mathrm{~S} \\
(437.58)\end{array}$ & $\begin{array}{ll}\mathrm{N} & 16.00 \\
\mathrm{~S} & 7.33\end{array}$ & $\begin{array}{r}15.80 \\
7.20\end{array}$ \\
\hline $\mathrm{c}$ & $-\mathrm{CH}_{2} \mathrm{C}_{6} \mathrm{H}_{5}$ & 88 & $\begin{array}{c}215-17 \\
\text { (A) }\end{array}$ & $\begin{array}{l}\mathrm{C}_{24} \mathrm{H}_{23} \mathrm{~N}_{5} \mathrm{O}_{2} \mathrm{~S} \\
(445.55)\end{array}$ & $\begin{array}{lr}\mathrm{C} & 64.70 \\
\mathrm{H} & 5.20 \\
\mathrm{~N} & 15.72\end{array}$ & $\begin{array}{r}64.99 \\
4.95 \\
15.99\end{array}$ \\
\hline $\mathrm{d}$ & $-\mathrm{C}_{6} \mathrm{H}_{5}$ & 73 & $\begin{array}{c}249-51 \\
\text { (B) }\end{array}$ & $\begin{array}{l}\mathrm{C}_{23} \mathrm{H}_{21} \mathrm{~N}_{5} \mathrm{O}_{2} \mathrm{~S} \\
(431.52)\end{array}$ & $\begin{array}{ll}\mathrm{N} & 16.23 \\
\mathrm{~S} & 7.43\end{array}$ & $\begin{array}{r}16.50 \\
7.50\end{array}$ \\
\hline $\mathrm{e}$ & $\begin{array}{l}-\mathrm{C}_{6} \mathrm{H}_{4} \mathrm{CH}_{3} \\
\text { (p) }\end{array}$ & 93 & $\begin{array}{l}206-8 \\
\text { (B) }\end{array}$ & $\begin{array}{l}\mathrm{C}_{24} \mathrm{H}_{23} \mathrm{~N}_{5} \mathrm{O}_{2} \mathrm{~S} \\
(445.55)\end{array}$ & $\begin{array}{ll}N & 15.72 \\
S & 7.20\end{array}$ & $\begin{array}{r}15.60 \\
7.30\end{array}$ \\
\hline
\end{tabular}

$\mathrm{A}=$ Ethanol, $\quad \mathrm{B}=$ Ethanol/water.

2-[4-(2-Substitutedamino-1,3,4-oxadiazol-5yl)phenoxymethyl]-1 $H$-benzimidazoles (IXa-e) and 2-[4-(2-substitutedamino-1,3,4-oxadiazol5-yl)phenoxymethyl]-1-methyl-1H-benzimidazoles (X a-e).

To a boiling stirred solution or suspension of the appropriate VII or VIII ( 0.001 mole) in ethanol $(30 \mathrm{ml})$, finely powdered yellow $\mathrm{HgO}$ $(0.2 \mathrm{~g}, 0.001$ mole) was added portion wise, over a period of $30 \mathrm{~min}$. The suspension was stirred and heated under reflux for 3-10 hours. The hot reaction mixture was filtered and the black precipitate was washed with boiling ethanol $(2 \mathrm{ml})$. The filtrate and washings were combined, concentrated to a small volume and treated with drops of water. The precipitate formed was filtered, dried and crystallized from the proper solvent (Table 4). The IR of compounds (IX a-e) $\left(\mathrm{cm}^{-1}\right)$ showed 3400-3200 $(\mathrm{NH})$, compounds (X a-e) showed 3440-3250 (NH). ${ }^{1} \mathrm{H}-\mathrm{NMR}$ of compound IX c (DMSO-d ${ }_{6}$ ) $\delta \mathrm{ppm} ; 4.56\left(\mathrm{~d}, 2 \mathrm{H}, \mathrm{J}=6 \mathrm{~Hz}, \mathrm{CH}_{2}-\mathrm{C}_{6}-\mathrm{H}_{5}\right), 5.52$ (s, 2H, $\left.\mathrm{CH}_{2}-\mathrm{O}\right), 7.36-8.08(\mathrm{~m}, 13 \mathrm{H}$, Ar- $-\underline{\mathrm{H}})$, 8.16 (br.s., 1H, N $\underline{H}$, deuterium exchangeable), 13 (br.s., $1 \mathrm{H}, \mathrm{N} \underline{\mathrm{H}}$, deuterium exchangeable). ${ }^{1} \mathrm{H}-\mathrm{NMR}$ of compound (X e) (DMSO-d $\left.{ }_{6}\right) \delta \mathrm{ppm}$; $2.4\left(\mathrm{~s}, 3 \mathrm{H}, \mathrm{C}_{6} \mathrm{H}_{5}-\mathrm{CH}_{3}\right), 4.0\left(\mathrm{~s}, 3 \mathrm{H}, \mathrm{N}-\mathrm{CH}_{3}\right)$, $5.76\left(\mathrm{~s}, 2 \mathrm{H}, \mathrm{CH}_{2}\right), 7.36-8.24(\mathrm{~m}, 12 \mathrm{H}, \mathrm{Ar}-\underline{\mathrm{H}})$, $10.96(\mathrm{~s}, 1 \mathrm{H}, \mathrm{N} \underline{\mathrm{H}})$, deuterium exchangeable).

\section{Antimicrobial Testing}

\section{A) Inhibition Zone Measurement}

Sterile Tryptic soy agar plates were prepared. The compounds to be tested were dissolved in dimethylformamide $(10 \mathrm{mg} / \mathrm{ml})$ and $20 \mu \mathrm{l}$ each of these solutions were then dropped over the agar medium, and allowed to dry. The plates were then overplayed with $10 \mathrm{ml}$ molten Tryptic soy agar inoculated with the test organism ( $20 \mu \mathrm{l}$ of 24 hours Tryptic soy broth culture). The plates were incubated at $37^{\circ}$ for 24 hours and the resulting inhibition zones were 
measured. A control without the test compound was included for each organism. Amikacin (30 $\mu \mathrm{g} / \mathrm{ml}$ ) was used as a broad spectrum reference standard against the tested microorganisms (inhibition zone $17 \mathrm{~mm}$. in diameter). Compounds which showed inhibition zones $\geq$ $17 \mathrm{~mm}$ in diameter were evaluated for their minimal inhibitory concentration (MIC).

\section{B) Minimal inhibitory concentration (MIC) Measurement}

The test organism was grown in Tryptic soy broth for 24 hours at $37^{\circ} \mathrm{C}$. The compound to be tested was dissolved in dimethylformamide (500 $\mu \mathrm{g} / \mathrm{ml}$ ) and two fold serial dilutions were prepared using Tryptic soy broth. The tubes were then inoculated with $100 \mu$ lof the 24 hours test organism culture and were incubated at $37^{\circ} \mathrm{C}$ for 48 hours. The tubes showing turbidity were subcultured on Tryptic soy agar plates to confirm the presence or absence of bacterial growth.

\section{RESULTS AND DISCUSSION}

\section{A- Chemistry}

The new series of compounds were designed and synthesized comprising substituted oxadizole moiety joined to a substituted benzimidazole ring. It was hoped that some of the substituted isomers would yield a potent antibacterial or antifungal agents. Two substituted acid hydrazide I and II were selected as starting materials for this purpose.

The 4-(1H-2-benzimidazolyl methyloxy) benzoic acid hydrazide (I) was prepared according to a reported method ${ }^{13}$ and the 4-(1methyl-1H-2-benzimidazolyl methyloxy) benzoic acid hydrazide (II) was prepared from 2-[4(ethoxycarbonyl)phenoxymethyl]-1-methyl-1 $H$ benzimidazole ${ }^{12}$ by reaction with hydrazine hydrate (Scheme 1). The 2-[4-(alkylidene or arylidenehydrazinocarbonyl)phenoxymethyl]-1Hbenzimidazoles (III a-f) and 2-[4-(alkylidene or arylidenehydrazinocarbonyl)phenoxymethyl]-1methyl-1H-benzimidazoles (IV a-e) were prepared by reaction of the acid hydrazide (I or II) with appropriate aldehydes or ketones.
Cyclization of compounds (III a-f, IV a-e) with acetic anhydride gave 2-[4-(3-acetyl-2substituted-2,3-dihydro-1,3,4-oxadiazole-5yl)phenoxymethyl]-1 $H$-benzimidazoles ( $\mathrm{V}$ a-f) and 2-[4-(3-acetyl-2-substituted-2,3-dihydro1,3,4-oxadiazol-5-yl)phenoxymethyl]-1-methyl$1 H$-benzimidazoles (VI a-e).

The 2-[4-(butyl, cyclohexyl, benzyl, phenyl or p-tolyl-thiocarbamoylhydrazinocarbonyl) phenoxymethyl]-1 $H$-benzimidazoles (VII) were prepared as reported ${ }^{14}$. The 2-[4-(substituted thiocarbamoylhydrazinocarbonyl)phenoxymeth yl]-1-methyl-1H-benzimidazoles (VIII a-e) were prepared by reaction of (II) with the appropriate isothiocyanates. Compounds (VII) and (VIII) were converted to 2-[4-(2-substitutedamino1,3,4-oxadiazol-5-yl)phenoxymethyl]-1 $H$-benzimidazoles (IX a-e) and 2-[4-(2-substitutedamino1,3,4-oxadiazol-5-yl)phenoxymethyl]-1-methyl$1 H$-benzimidazoles (X a-e) by cyclodesulfurization with freshly prepared yellow mercuric oxide.

The structures of the products were substantiated by microanalyses, IR and ' $\mathrm{H}-\mathrm{NMR}$ of representative examples.

\section{B- Antimicrobial Activity}

The newly prepared compounds were tested for in vitro activity against two Gram-negative bacilli (E. coli, K. pneumonia), Gram-positive cocci (Staphylococcus aureus: Oxford strain and penicillinase producer) and single strain of Candida albicans, using the agar diffusion method $^{15}$. Amikacin $(30 \mu \mathrm{g} / \mathrm{ml})$ and $0.01 \%$ solution of nystatin were used as a reference standards (Table 5).

All the compounds showed no antibacterial potency (inhibition zone $<17 \mathrm{~mm}$ in diameter) against the tested Gram-negative bacilli and Gram-positive cocci except compound (IX e) which gave an inhibition zone of $22 \mathrm{~mm}$ and 20 $\mathrm{mm}$ in diameter to Gram-negative bacilli and Gram-positive cocci respectively, MIC was $15.625(\mu \mathrm{g} / \mathrm{ml})$. All the compounds were also found to lack the antifungal activity against the tested fungus (a single strain of Candida albicans). 
Table 4: 2-[4-(2-Substitutedamino-1,3,4-oxadiazol-5-yl)phenoxymethyl]-1 H-benzimidazoles (IXae) and 2-[4-(2-substituted amino-1,3,4-oxadiazol-5-yl)phenoxymethyl]-1-methyl-1 $H$ benzimidazoles ( $\mathrm{X}$ a-e).

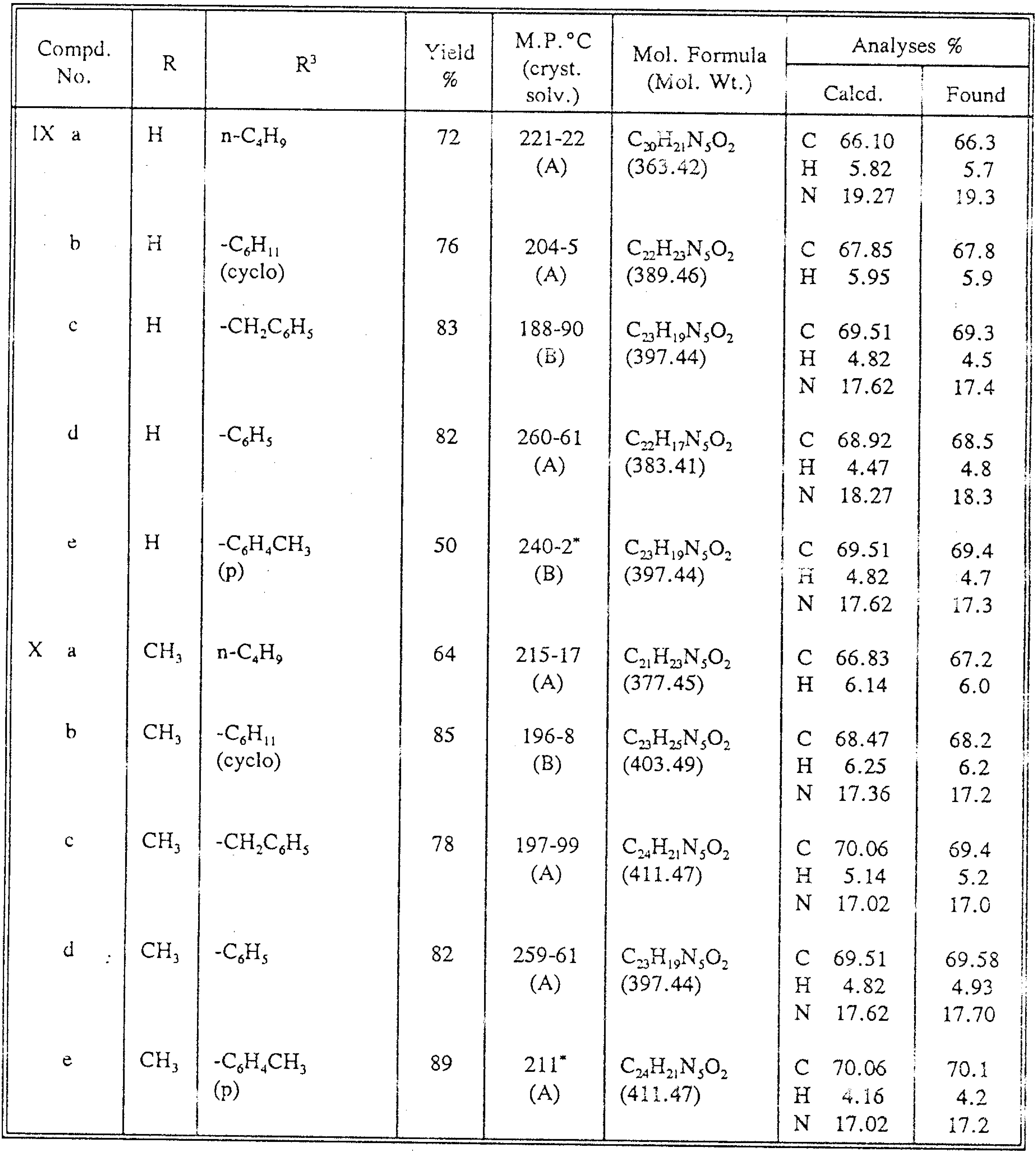

$A=$ Ethanol, $\quad B=$ Ethanol/water.

* Melting with decomposition. 
Table 5: $\quad$ Growth inhibition zones (mm) exhibited by the newly prepared compounds.

\begin{tabular}{|c|c|c|c|c|c|c|}
\hline \multirow{2}{*}{\multicolumn{2}{|c|}{ Compd. }} & \multicolumn{2}{|c|}{ S. aureus } & \multirow{2}{*}{ E. Coli } & \multirow[b]{2}{*}{ K. Pneumonia } & \multirow[b]{2}{*}{ C. albicans } \\
\hline & & Oxford & $\begin{array}{l}\text { Penicillinase } \\
\text { Producer } \\
\end{array}$ & & & \\
\hline \multicolumn{2}{|l|}{ II } & - & - & - & - & - \\
\hline \multirow[t]{5}{*}{ III } & $\mathbf{a}$ & 13 & - & - & - & - \\
\hline & b & - & - & - & - & - \\
\hline & $\begin{array}{l}c \\
\text { d }\end{array}$ & - & - & - & - & - \\
\hline & e & - & - & - & - & - \\
\hline & f & - & - & - & - & - \\
\hline \multirow[t]{4}{*}{ IV } & a & - & - & - & - & - \\
\hline & b & - & - & - & - & - \\
\hline & $\begin{array}{l}c \\
d\end{array}$ & - & - & - & - & - \\
\hline & e & - & - & - & - & - \\
\hline \multirow[t]{5}{*}{ V } & $\mathbf{a}$ & - & - & $\cdots$ & - & - \\
\hline & b & - & - & - & - & - \\
\hline & c & - & - & - & - & - \\
\hline & $\begin{array}{l}d \\
e\end{array}$ & - & - & - & - & - \\
\hline & f & - & - & - & - & - \\
\hline \multirow[t]{4}{*}{ VI } & $\mathbf{a}$ & - & - & - & - & - \\
\hline & b & $\overline{9}$ & - & - & - & - \\
\hline & $\begin{array}{l}c \\
d\end{array}$ & $\begin{array}{l}9 \\
-\end{array}$ & 10 & - & - & - \\
\hline & e & - & - & - & - & - \\
\hline \multirow[t]{4}{*}{ VIII } & $\mathbf{a}$ & 16 & 14 & - & - & - \\
\hline & b & - & - & - & - & - \\
\hline & $\begin{array}{l}c \\
d\end{array}$ & - & - & - & - & - \\
\hline & e & - & - & - & - & - \\
\hline \multirow[t]{4}{*}{ IX } & $\mathbf{a}$ & - & - & - & - & - \\
\hline & b & - & $\therefore$ & - & - & - \\
\hline & $\begin{array}{c}c \\
d\end{array}$ & $\begin{array}{c}10 \\
-\end{array}$ & $\begin{array}{l}11 \\
-\end{array}$ & 12 & - & - \\
\hline & $e^{*}$ & 20 & 20 & 22 & 22 & - \\
\hline \multirow[t]{4}{*}{$\mathrm{X}$} & $\mathbf{a}$ & - & - & - & - & - \\
\hline & b & - & - & - & - & - \\
\hline & $\begin{array}{c}c \\
d\end{array}$ & - & - & - & - & - \\
\hline & e & - & - & - & - & - \\
\hline \multicolumn{2}{|c|}{$\begin{array}{l}\text { Amikacin } \\
(30 \mu / \mathrm{ml})\end{array}$} & 17 & 17 & 17 & 17 & - \\
\hline \multicolumn{2}{|c|}{$\begin{array}{l}\text { Nystatin } \\
(0.01 \%)\end{array}$} & - & - & - & - & 22 \\
\hline
\end{tabular}




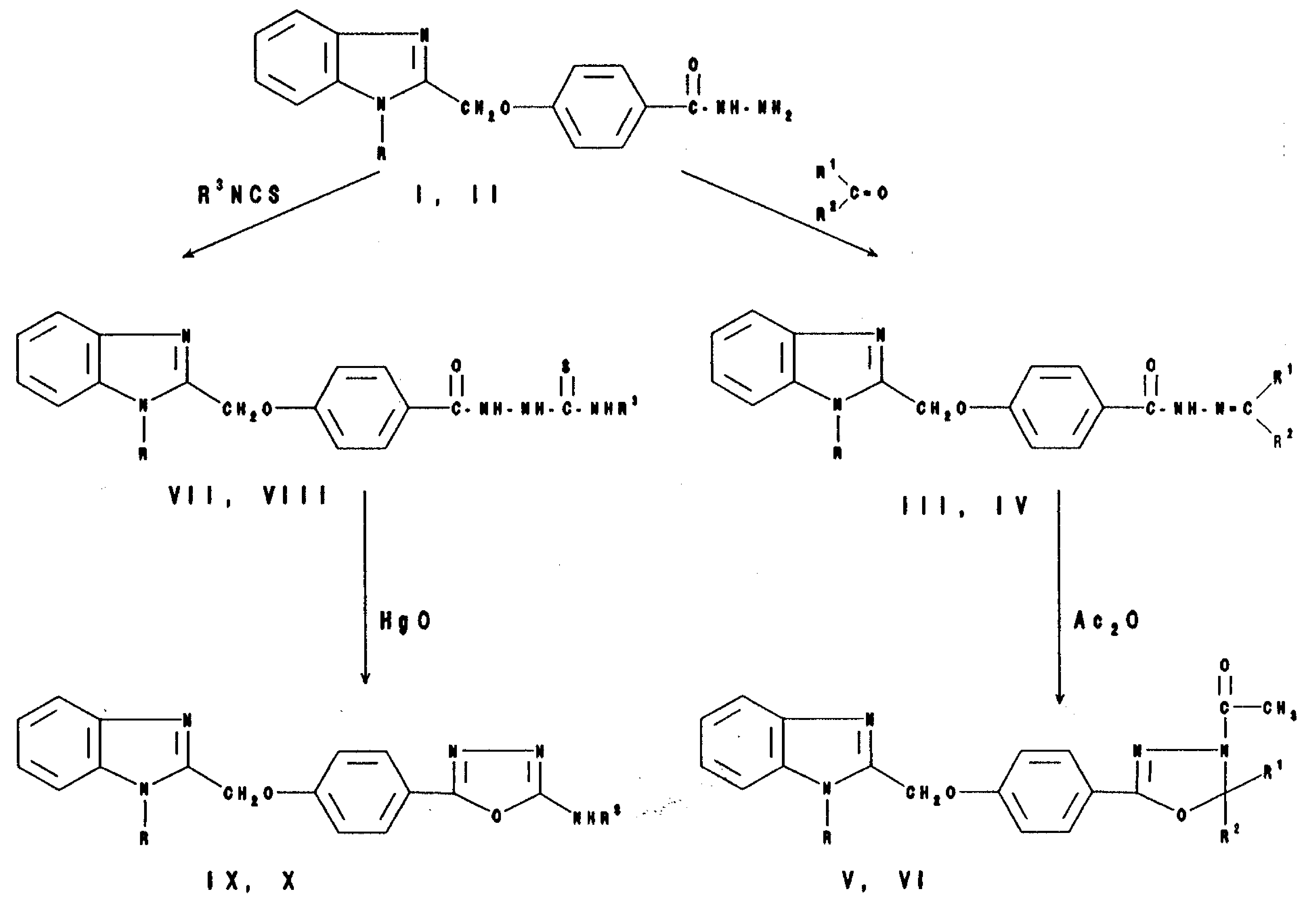

Scheme 1

\section{REFERENCES}

1. A.K.Sengupata and M.J.Garg, Antibact. Antifung. Agents. 9, 397 (1981).

2. K.H.Sinnur, S.Siddappa, Shivagogi, R.Purochit and G.Muralidhar, Indian J. Chem. Sect. B. 25B, 716 (1986).

3. K.P.Roda, R.N.Vansdadia, Parekh, Hansa, J. Indian Chem. Soc. 65, 44 (1988).

4. R.S.Sharma and S.C.Bahel, J. Antibact. Antifung. Agents, 10, 294 (1982).

5. H. Singh, A.R.Misra and L.D.S.Yadav, Indian J. Chem. Sect. B. 26B, 1000 (1987).

6. M.M.Dulta, B.N.Goswami and J.C.S.Kptaky, J. Indian Chem. Soc. 64, 195 (1987).

7. G.Heywang, B.Baasner, G.Haenssier, W.Paulys, H.J.Santel, R.R.Schmidt and H.G.Schmitt, Ger.Offen (1988) De 3, 621, 265 C.A., 108, 221702f (1988).
8. S.Bahadur, M.Sazena and K.K. Pandey, J. Indian Chem. Soc., 58, 1018 (1981).

9. V.K.Mishra and S.C.Bahel, Indian J. Pharm. Sci., 47, 5 (1985).

10. A.C.Baillie, A.D.Buss, J.H.Parsons and P.E.Russel, Eur. Pat. EP 219, 192 (1987), C.A. 107, 134305g (1987).

11. F.A.Ashour and S.A.Al-Mazroa, Alex. J. Pharm. Sci., 4, 29 (1990).

12. F.A.Ashour, Alex. J. Pharm. Sci., 5, 16 (1991).

13. S.Bahadur and K.K.Pandy, Pharmazie, 34 , 570 (1979).

14. F.A.Ashour and S.A.Al-Mazroa, IL Farmaco, 45, 1207 (1990).

15. J.E.Conti and S.L.Barriere "Manual antibiotics and infectious diseases" 1st. ed. Copyright, Leo and Febiger U.S.A. (1985). 